Article

Covid 19 and Diet Pattern

Journal of Development Economics and

Management Research Studies (JDMS), A Peer Reviewed Open Access

International Journal

ISSN: 25825119 (Online)

Crossref Prefix No: 10.53422

08(09), 51-54, July-September, 2021

@ Center for Development Economic Studies

(CDES)

Reprints and permissions

http://www.cdes.org.in/

http://www.cdes.org.in/journal/

\title{
Covid 19 and Diet Pattern
}

\section{Dr.R.Sivachandran ${ }^{1}$ and Dr.K.Rajagopal ${ }^{2}$}

\begin{abstract}
COVID-19 causes problems like cytokine release syndrome or cytokine storm and the infection triggers the immune system to flood bloodstream with inflammatory proteins called cytokines. It kills tissue and damage organs. The only sustainable way to survive from the current situation is to strengthen the immune system. An adequate intake of zinc, iron, and vitamins $A, B 12, B 6, C$, and $E$ is essential for the maintenance of a good immune function. $A$ balanced diet will guarantee a strong immune system that can help to withstand the effect of a viral attack.
\end{abstract}

Keywords: COVID-19, immune, respiratory tracts, cytokines, Thirumoolar.

\section{Introduction}

The Coronavirus is a unique virus that causes an infection in the respiratory tracts like the nose, sinuses, or upper throat. In early 2020, after December 2019 the outbreak in China, the World Health Organization identified SARS-CoV-2 as a new type of coronavirus. The epidemic rapidly spread around the world and become pandemic. The COVID-19 is a disease caused by SARS-CoV-2 that can cause what doctors call a respiratory tract infection. It can affect person's upper respiratory tract. It blowouts the same way other coronaviruses do, mainly through person-to-person contact. Infections range from mild to deadly. SARS-CoV-2 is one of seven types of coronavirus, including the ones that cause severe diseases like the Middle East Respiratory Syndrome (MERS) and unexpected Severe Acute Respiratory Syndrome (SARS). The other coronaviruses cause most of the colds that affect us during the year but are not a serious threat for otherwise healthy people. The key symptoms include Fever, Coughing, Shortness of breath, trouble breathing, Fatigue, Chills, sometimes with shaking, Body aches, Headache, Sore throat, Congestion/runny nose, loss of smell or taste,

\footnotetext{
${ }^{1}$ Assistant Professor of Zoology, RKM Vivekananda College (Autonomous), Mylapore, Chennai 600004.

${ }^{2}$ Assistant Professor of Botany, RKM Vivekananda College (Autonomous), Mylapore, Chennai 600004.
} 
Nausea and Diarrhea. The virus can lead to pneumonia, respiratory failure, heart problems, liver problems, septic shock, and death. Many COVID-19 complications may be caused by a condition known as cytokine release syndrome or a cytokine storm. This is when an infection triggers immune system to flood bloodstream with inflammatory proteins called cytokines. They can kill tissue and damage your organs.

While it is vital to mention hygiene, standards like washing your hands frequently, especially if you have travelled by public transport and after visit to crowded market place. Using an alcohol sanitizer, in case you are travelling to disinfect your hands, wearing a mask (cover your nose and mouth) and avoiding touching your hand or mouth. There are also certain methods to improve your immunity, which is paramount now. The food you eat plays a key aspect in determining your overall health and immunity.

More than 2,500 years ago, Hippocrates said: "Let food be thy medicine and medicine be thy food." Thirumoolar also have said (உணவே மருந்து) Unave Marunthu. Both food intake and incidence of the disease usually influence the nutritional status of people, particularly in a developing nation, where everyone is striving for food. Insufficient diet and infectious diseases can lead to severe malnutrition.

Currently, the COVID-19 pandemic is the prominent challenge across the globe, therefore scientists and researchers are endeavouring to create a specific vaccine for this virus. Even if vaccines discovered, there is a high possibility that other antimicrobial impervious infections will prevail. Intake of nutritional food is very essential to maintain a strong immune system against any disease causing pathogen.

At a time when the world is battling with the Coronavirus widespread, and with no remedy or immunization available, the best we could do is to extend our body's protections against the illness. A normal workout schedule and an appropriate diet is exceptionally vital to battle the infection.

Certain aspects such as lifestyle, age, sex, and medications affect the nutritional status of an individual. During the COVID-19 pandemic, the nutritional status of individual is used as a measure of resilience toward destabilization. In addition, different dietary ingredients are determinants of gut microbial composition and consequently shape the immune responses in the body. Therefore, the existing evidence proposes that the only sustainable way to survive in the current situation is to strengthen the immune system. An adequate intake of zinc, iron, and vitamins A, B 12, B6, C, and E is essential for the maintenance of a good immune function. COVID-19 has forced a new set of challenges for the individual to maintain a hale and healthy diet. The state of self-isolation, lockdown, and social distancing are important measures to flattening the curve of the disease, although these measures have severe consequences on an individual's life. The act of confining to one's home has significant impacts on one's health, including changes in eating patterns, sleeping habits, and physical activity. It would promote sedentary behaviours that affect mental and physical health and lead to an increased risk of obesity. Fear and anxiety may cause changes in dietary habits leading to unhealthy dietary patterns and less desire to eat.

In any case, the stationary way of life and off base eating propensities have been the greatest obstacle in building a solid resistance against pathogenic infection. One of the most important ways to boost our immune is by including natural products and vegetables in our diet that will provide our body the correct dosage of proteins and vitamins.

A balanced diet will guarantee a strong immune system that can help to withstand the effect of a viral attack. There is currently no scientific evidence that any supplement can 'boost' our immune system, except Vitamin C. Vitamin C is one of the major constituents of water-soluble vitamins, which tends to make a strong immune system. The daily- 
recommended dietary allowance for Vitamin C is $90 \mathrm{mg} / \mathrm{d}$ for men and $75 \mathrm{mg} / \mathrm{d}$ for women. In the current situation, it is necessary to be aware of the specific types of food that can improve our immune system in order to combat COVID-19. Here are some professional and authentic dietary guidelines to resist COVID-19 infection.

Vitamin $\mathrm{A}$ is an anti-inflammatory vitamin and plays a basic part in improving resistance. Beta-carotene could be an antecedent of vitamin A which when ingested gets a changeover within the digestive system to vitamin A. Sources of beta carotenecarrots, sweet potatoes, ruddy and yellow peppers, tomatoes, dim green verdant vegetable, pumpkins, asparagus, mangoes, drumstick leaves.

Vitamin B6 plays an imperative part in supporting the biochemical response of the resistant system. Sources: poultry, nuts, chickpeas, dim green leafy veggies, bananas, and papayas.

Vitamin B12 could be a crucial supplement for the sound production of blood cells and DNA union. Vitamin B12 together with folic acid plays a pivotal part in keeping up a solid safe system. Sources: meat, poultry, eggs, grain, and grain products.

Vitamin $\mathrm{C}$ is the foremost antioxidant known to boost insusceptibility. It also makes a difference in the recovery of Vitamin E. Sources: Indian gooseberry (amla), guava, orange.

Vitamin D is basic to safe functioning of our body and makes a difference in directing the body's safe reaction. We can get Vitamin D by exposing our body in daylight either or overhang without sunscreen amid the day ideally between 11 a.m. and 1 p.m. Sources: Egg Yolk, cod liver oil, mushrooms, sardines, and fatty fish.

Vitamin $\mathrm{E}$ is a fat-soluble vitamin that plays a key part in controlling and supporting immune framework. It avoids oxidation of Beta Carotene and Vitamin A within the intestine. Sources: nuts such as almonds, pistachios, seeds like sunflower seeds, flax seeds.

In expansion to protein and vitamins, certain minerals like zinc, magnesium, selenium together with omega 3 fats play a key part in boosting immunity. Seafood is a nutrient rich food that reduces inflammation with vitamins A, B, and D, as well as omega-3 fatty acids and minerals such as calcium, selenium, phosphorus, iron, zinc, iodine, magnesium and potassium. Protein is a versatile macronutrient that supports life and plays a vital role in boosting our immune system. In spite of the fact that we all know the significance of protein, Indians are lacking in proper protein intake. A normal person needs $0.8-1.0 \mathrm{~g}$ of protein per $\mathrm{kg}$ of body weight. Suggestions may shift depending on a few variables such as physical action, age, co dismal conditions like kidney illness etc. As per the Common Buyer Overview (PODIGY) conducted in 2015, 73 percent of our population is lacking in protein admissions with 93 percent of our general public being uninformed about their everyday protein requirements. It is important to intake appropriate and good quality of protein everyday for the safe framework and operation of our body at its best. A quarter of our plate at each dinner must be protein. A great quality to begin with course protein is the one with high digestibility and has all the fundamental amino acids required by the body. Best sources could be egg, meat and fish.

Good nutrition is essential to support a strong immune system, which may offer protection from many health problems including chronic diseases. The Dietary Guidelines recommend regular fish and seafood consumption for optimal wellness of the body. Data from the NIH-AARP Diet and Health Study of more than 420,000 people shows regular intake of seafood reduced respiratory deaths by 20 percent. Everyone can benefit from eating the recommended amount of seafood. Seafood is a great option to include on your weekly 
diet menu because it pairs well with other food groups. Eating a balanced diet of fruits, vegetables, whole grains, legumes, seafood and fatty fish, lean meats, low-fat dairy products, and nuts and seeds helps in safe functioning of our body. All these foods have a variety of key nutrients that contribute to immune health. If one isn't getting an adequate amount of these foods in their diet daily.

Other lifestyle habits important to health and supporting the immune system are exercise, getting adequate sleep and managing stress. We can provide our resistant framework a boost by including anti-viral nourishments effortlessly accessible in our kitchen like garlic, fennel, ginger, cloves, tulsi, turmeric and coconut oil. If we make straightforward tweaks to count calories by counting immune boosting supplements to assist to battle with the contamination. Finally, do not ignore to preserve cleanliness by washing hands routinely, when in public gathering or public place keep up physical distancing.

\section{REFERENCES}

1. Bogoch II, Watts A, Thomas-Bachli A, Huber C, Kraemer MU, Khan K. Pneumonia of unknown etiology in Wuhan, China: potential for international spread via commercial air travel. J Travel Med.2020;272:1-3. [PMC free article] [PubMed] [Google Scholar]

2. Yousafzai AK, Rasheed MA, Bhutta ZA. Annual research review: improved nutrition-a pathway to resilience. J Child Psychol Psychiatry. 2013;54:367377. [PubMed] [Google Scholar]

3. Ksiazek TG, Erdman D, Goldsmith CS, Zaki SR, Peret T. Emery S, Tong S, et al., editors. A novel coronavirus associated with severe acute respiratory syndrome. $\mathrm{N}$ Engl J Med. 2003;348(20):1953-1966. [PubMed] [Google Scholar]

4. Anton SD, Miller PM. Do negative emotions predict alcohol consumption, saturated fat intake, and physical activity in older adults? BehavModif. 2005;29:677688. [PubMed] [Google Scholar]

5. Aslam MF, Majeed S, Aslam S, Irfan JA. Vitamins: Key role players in boosting up immune response, A mini review Vitam. Miner. 2017;6:153. [Google Scholar]

6. Macht M. How emotions affect eating:a five-way model. Appetite. 2008;50:111. [PubMed] [Google Scholar]

7. Gleeson M, Nieman DC, Pedersen BK. Exercise, nutrition and immune function. $\mathbf{J}$ Sports Sci. 2004;22:115-125. [PubMed] [Google Scholar]

8. Childs C. et al. Diet and Immune Function. Nutrients. 2019 Aug; 11(8): 1933. https://www.ncbi.nlm.nih.gov/pmc/articles/PMC6723551/

9. U.S. Department of Health and Human Services and U.S. Department of Agriculture. 2015 - 2020 Dietary Guidelines for Americans. https://www.dietaryguidelines.gov/

10. Zhang, Y. et al. Association of fish and long-chain omega-3 fatty acids intakes with total and cause-specific mortality: prospective analysis of 421309 individuals. J Intern Med. 2018 Oct;284(4):399-417.

11. Gleeson M, Nieman DC, Pedersen BK. Exercise, nutrition and immune function. J Sports Sci. 2004;22:115-125. [PubMed] [Google Scholar]

12. Macht M. How emotions affect eating:a five-way model. Appetite. 2008;50:111. [PubMed] [Google Scholar] 